\title{
AVAILABILITY ASSESSMENT METHODS AND THEIR APPLICATION IN PRACTICE
}

\author{
Rommert Dekker ${ }^{1}$ and Wim Groenendijk ${ }^{2}$
}

\begin{abstract}
In this paper we compare availability assessment methods for continuous production systems in a design phase. Apart from discussing simulation and analytical techniques, we also consider computer tools and data sources. The comparison is based on a literature search, analytical considerations and on practical experience with the methods and the tools. Most progress in the last ten years seems to have been made on simulation programs and on databases, such as OREDA. This is somewhat disappointing, compared with the progress made in a related area.
\end{abstract}

\section{Introduction}

In this paper we consider methods for availability and effectiveness assessment of continuous production systems in a design phase, where the main objective is economic optimisation. That is, we deal with sizing and standby options of units in a design phase to determine whether the system design meets its requirements. Such analyses are common in for example the oil and gas industry. The present trend is to make the development of small oil and gas fields economically interesting by taking a 'minimum facilities' approach, i.e., by installing as little equipment as possible. This implies that designs should be critically evaluated on their effectiveness.

Numerous availability models have been published over the years, some analysed by simulation, but most by analytical techniques. Yet the general opinion some years ago was that analytical techniques were far too restrictive to tackle practical problems. In this paper we therefore

1 Erasmus University Rotterdam

2 Shell Internationale Petroleum Maatschappij B.V. 
review the developments over the last ten years. Especially we consider the question whether newly developed analytical techniques are able to tackle real problems and how they compare with simulation methods in actual practice.

The last review of availability assessment models is from Lie, Hwang and Tillman (1977). They list some 100 theoretical papers, dealing with various aspects, but very few with actual applications. Since that time many more papers have been published, but only on simple systems. Practical production systems usually consist of more than ten units and have complex aspects. Case studies published so far either deal with isolated or limited aspects of practical systems, or applied simulation.

The structure of this paper is as follows. After introducing aspects of the general availability problem we discuss the state-of-the-art for analytical techniques and simulation in section three. Section four deals with computer tools available and with the attempts to set up databases. The next section deals with practical experiences with availability assessment. The last section lists a number of problems and opportunities which come forward from practical experience.

\section{Availability assessment for oil and gas producing systems}

Oil and gas producing systems tend to have a relatively simple structure from a reliability viewpoint. They often consist of parallel trains of units, possibly with standby units and can therefore be modelled as a seriesparallel configuration. Availability assessment usually occurs at a unit level, (i.e. pumps, compressors etc.) and not at a component level as that is too detailed for the type of decision support that is required. The following figure gives a (simplified) example of such a system, a sea-water injection facility.

The main product in this system is of course the sea water. One of the by-products is formed by the injected chemicals. The two sea-water 


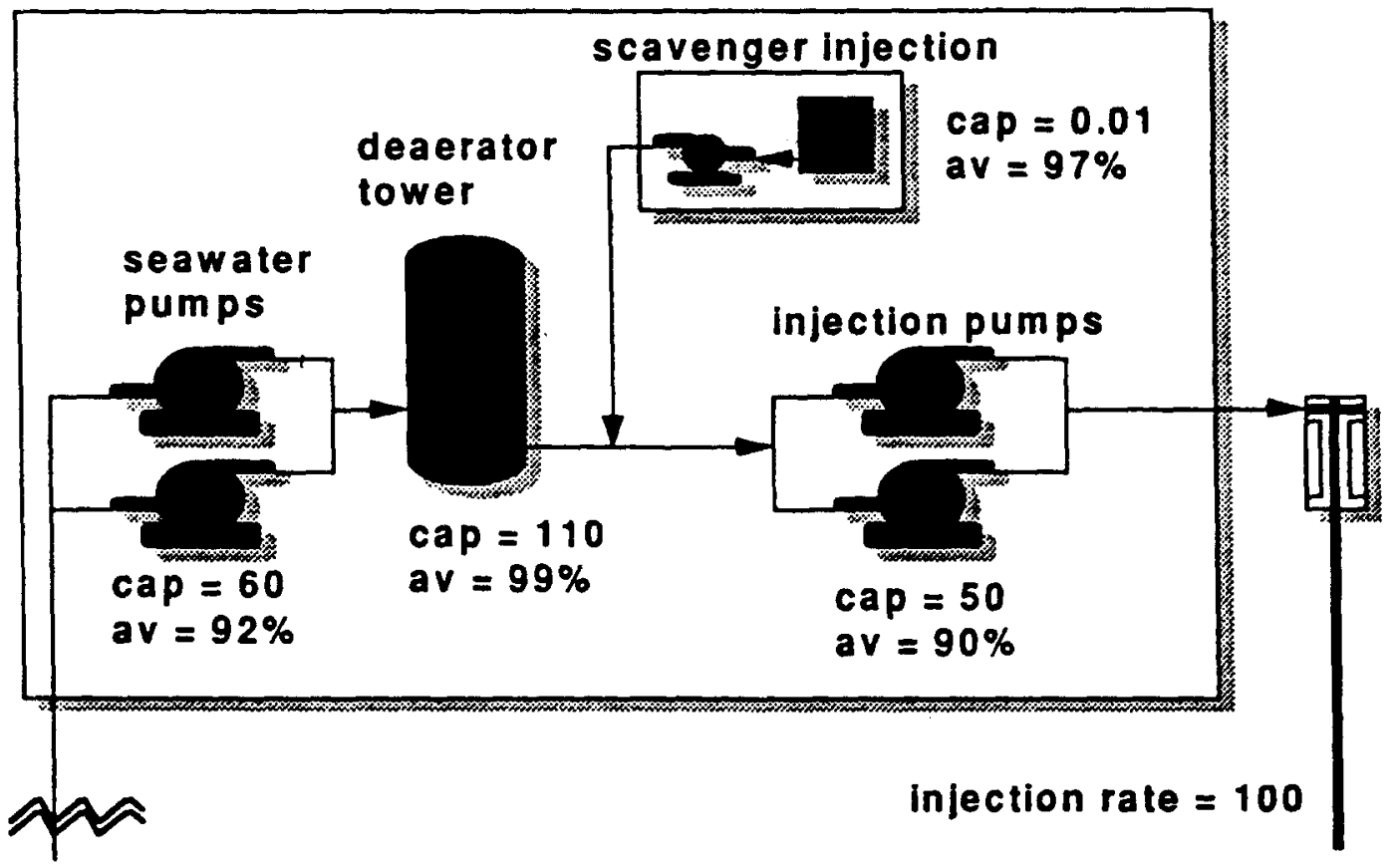

Figure 1: Sea-water injection facility

pumps each have a capacity of 60 ; together they provide the demanded rate of 100 . If one of the sea-water pumps breaks down the other is still capable of providing 60 . The pumps are therefore modelled in parallel. If the deaerator breaks down the facility is shut down; therefore the deaerator tower is modelled in series with the rest of the system. Similarly, if scavenger injection breaks down the facility is shut down; therefore scavenger injection is modelled in series with the rest of the system. Its capacity is set at some arbitrary, but high enough value since it is assumed that only its reliability influences the operation of the system, not its physical capacity. Finally, the two injection pumps are modelled in parallel, since each can provide $50 \%$ of the required capacity; if one injection pump breaks down, the other still supplies $50 \%$ of demand. The Reliability Block Diagram (RBD) for the water injection facility has been depicted below.

Complicating aspects in the modelling concern the various operating strategies, such as running with hot, warm or cold standby's. 


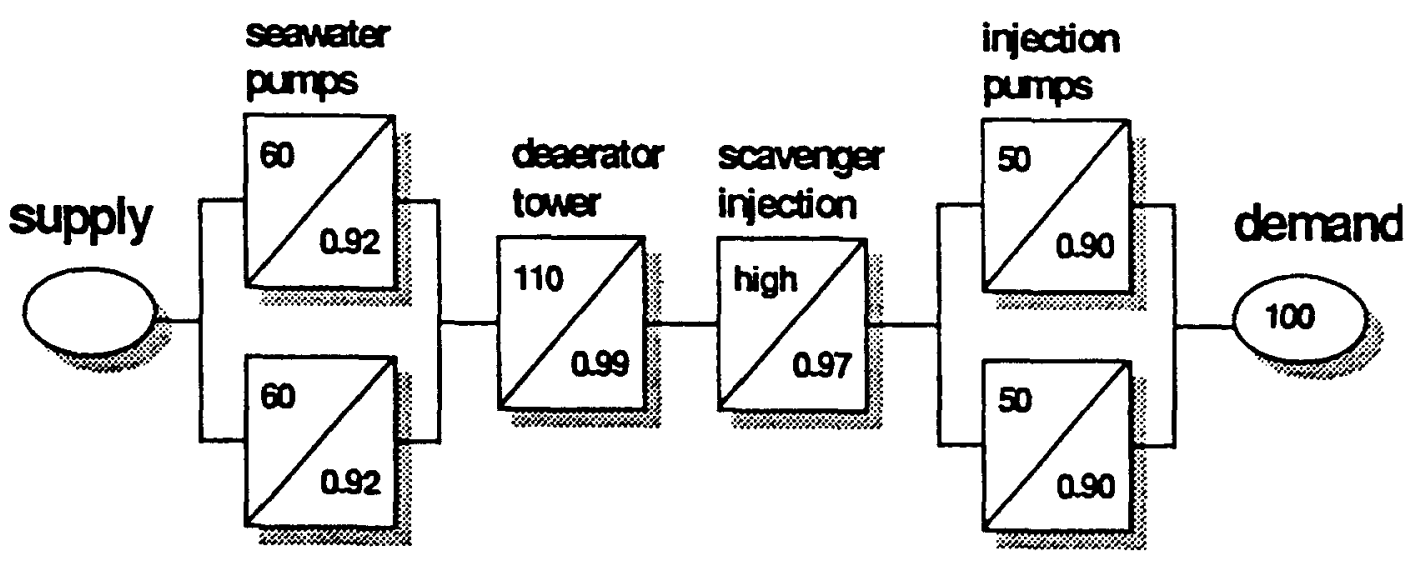

Figure 2: $\quad$ Sea-water injection facility RBD

Breakdowns may have several effects: production may be blocked or a bypass may be possible. Furthermore, in case of over capacity one has to decide on the load allocation and when and how much to boost. Intermediate storage poses another complication. The time to failure and repair for individual units may be taken as a (time-independent) exponential distributions. In more complex cases the distributions are more appropriately represented by Weibull, lognormal or others; furthermore, repairs may restore the condition only partially. Availability can be improved by preventive maintenance, which on the other hand also induces (planned) downtime. Condition monitoring techniques may also cause for intricate dependencies between the physical state of equipment and the timing of corrective maintenance, etc. Performance requirements may include transient statistics such as availability over a specified interval in time. Finally, various logistical aspects, such as unmanned operation also influence availability: It will be clear that the general availability assessment problem is not easy. A real example is given in section 5 .

\section{Methods for availability assessment}

Basically, there are two types of methods for determining the availability and effectiveness of a system, viz. Monte-Carlo simulation and analytical 
techniques. Both require a mathematical modelling of the system in terms of random variables for the state of the underlying units. Monte-Carlo simulation draws a realisation of each random variable and then determines which units are down and for how long, from which the system availability over the interval of interest can be determined. By repeating this procedure an estimate of the system availability is obtained. Analytical techniques, on the other hand, use structural results from applied probability theory to make statements on various performance measures, such as the steady-state or the interval availability. Below we briefly discuss the major developments in both areas in the last decade.

\subsection{Simulation}

The most eye-catching developments with respect to simulation were in the software area. Both object-oriented simulation packages and the animation possibility are the major improvements today. The few developments on the technique side got less attention. We like to mention variance reduction techniques, like importance sampling, but also the possibility for derivative estimation, which is useful for optimization (see e.g. Schmeiser (1990) for a recent overview). In our opinion there is significant scope for implementation of such developments in the available simulation software for availability analysis.

\subsection{Analytical methods}

On the analytical side of availability assessment few developments in the last decade are worth mentioning. Still many papers are submitted which analyse models with techniques from the sixties and seventies. These techniques are nicely summarised in Lie, Hwang and Tillman (1977) and in Birolini (1985). The latter gives a nice overview of a number of simple models which can be tackled analytically. Judging from published results in this area, however, these models cannot easily be expanded to model 
realistic situations. Techniques are the Markov models with exponential residence times and the semi-Markov models which are analysed by taking Laplace transforms for determining average performance measures. It is surprising that apart from some exceptions there have not been major breakthroughs, as in related fields it has been the case. For example, a related subject, performability analysis of computer and telecommunication systems has been a fruitful area in the last decade. Product form solutions, mean value analysis and various approximative methods are some of the major developments worth mentioning. Researchers in these directions mainly come from queueing theory or computer science, not from reliability engineering.

There are, however, some exceptions worth mentioning. Shell Research has been active on research in availability modelling. One major result is an aggregation technique with which realistic, large production networks can be analysed (see Van der Heiden and Schornagel (1988), Van Rijn and Schornagel (1987) and Brouwers (1986)/. In our opinion this research has finally led to methods with which realistic systems can be analysed, including features such as storage units and analysis of the effectiveness over (finite-length) contract periods.

\section{Decision support systems for availability assessment}

An essential pre-requisite for performing an availability study is not only to have a technique ready, but also a tool to apply it. As these techniques are to be applied in an industrial environment, the study usually has to be performed in a limited amount of time. Hence it is essential that a good computer system supports the analysis.

\subsection{Generic models}

An important element in the development of a decision support system (dss) is the modelling capability. That is, the selection of systems that 
can be tackled by the dss. One can not expect that a user is able to change software to include his specific model, therefore it is very important to describe on forehand which systems can be tackled and which cannot. In case of analytic tools the modelling is restricted to those aspects that can be dealt with analytically. In case of simulation, the choice is much wider, but also here it is important not to introduce too much complexity. The k-out-of-n subsystem, the series system and the single unit with a storage facility seem to be such general elements. Combined with an aggregation technique which replaces such an element by a unit with the same availability characteristics, now allows an analysis of all systems which can be built up of these generic elements.

\subsection{Software means}

Three aspects are of importance with respect to software. The user interface for modelling and result explanation, the analysis tools and finally the databases. Any software which is not used on a daily basis should be very userfriendly in order to remain used. A graphical interface for defining the system structure seems to be a preferred, but yet hardly realised way. A complication especially with simulation programs, is that the systems are analysed in such detail that it becomes extremely difficult to oversee the main aspects. To our knowledge no simulation programs is explaining its results, or indicating which input is essential and which is not.

Simulation is based on drawing random numbers many times. Too many details entered into simulation programs lead to excessive run times and data input times. Although the analytical programs have the disadvantage of being less general, they limit the data input and are generally much faster in computation. The rigid interface imposed by the analytical programs forces the user to think about the modelling aspects of the system in advance, which could actually be considered an advantage. Another advantage of analytical programs over simulation is 
that the former can be methodically tested and verified, which is an important factor to establish confidence in the results generated. Monte-Carlo simulation programs are notoriously difficult to verify for obvious reasons.

Finally, it is important that generic reliability data to feed the availability software is available on-line in a standardized format. Recent years have seen major steps forward in this area.

\subsection{Actual packages}

As a common observation, the development of software for availability assessment requires a major effort. The PC environment seems to be favoured for running these programs due to its easy accessibility and ease of use. Workstations lack a standard, especially for dealing with graphics, and are more complex. In the typical environment in which these programs are used PC's are much more widely spread.

\subsubsection{Availability assessment software}

The vast majority of availability assessment software is based on Monte-Carlo simulation. We mention the following, commercially available, packages: MIRIAM $^{3}$

MAROS $^{4}$

RAMP 5

PC-FOSP6

EDS-Scicon, Wavendon Tower, Wavendon, Milton Keynesm Bucks. MK 17 BLX, UK

4 Baker Jardine, Whitworth Building, Nat. Engin. Lab., East Kilbride, Glasgow G75 0QU, Scotland.

5 TA Consultancy Services Ltd., 'Newnhams', West Street, Farnham, Surrey, GU9 7EQ, UK.

$6 \quad$ Sintef Safety and Reliability, N-7034 Trondheim, Norway. 
PC-FOSP is somewhat different from the first three programs in the sense that it is specifically oriented towards sub-sea developments. All programs can run in a PC environment. An important aspect with these programs is the support from the supplier. Very often these programs are used by the suppliers acting as consultants, performing the work on a turn-key basis. Therefore the quality of the support provided by the suppliers is a major factor in selecting a software program for an availability analysis.

To our knowledge, the $\operatorname{RAMA}^{7}$ program is the only analytical availability assessment program on the market. Amongst others, it incorporates a graphical interface allowing on-screen model building. Within Shell, the in-house developed CREDO software is used for availability studies. CREDO is based on mathematical algorithms described in Van der Heijden and Schornagel (1988). Due to the limitations in modelling flexibility the analytical programs will be most useful in the front-end engineering phase of projects, when relatively little detailed information is available and uncertainties are still large. The simulation programs may be better suited to model highly detailed operational strategies and maintenance routines.

\subsubsection{Reliability databases - The OREDA project}

The availability of appropriate data for performing availability studies has traditionally been a problem. Many Maintenance Management Systems (MMS) in use in the industry cannot provide the type and quality of information required for such a study. In recent years this situation has improved significantly due to the establishment of the OREDA OOffshore REliability DAta) database (see OREDA (1992)). The OREDA project is a Joint Industry Project between ten major oil companies. The database 
contains data on approximately 7600 equipment items and 11,500 failure events for several types of offshore equipment. Additionally, the preventive maintenance programme for each equipment is recorded as well as the design data and operating conditions lincluding data on manufacturer, etc.). The database can be run on any IBM compatible PC. The generic data from OREDA can conveniently be used as a starting point for availability analyses. One of the most significant aspects of the OREDA project concerns the standardisation of reliability data format, terminology and data collection procedures. Preparations are being made to propose the OREDA format and data collection guide-lines as an ISO standard. Future plans with OREDA include expansion to onshore equipment, and manufacturing and chemical industry

\section{Practical experiences}

In this section a simplified example is presented of a typical availability analysis, demonstrating how such systems can be modelled and analysed. The message of this section is that the system presented can still quite easily be modelled with the analytical programs described in Section 4. No data nor specific results are given because they are not important for our message, moreover, they would require too much space.

\subsection{Description of the process system}

This study could be part of the conceptual design phase of a process plant. The objectives of the analysis could for instance be:

to establish minimum equipment level to meet specified demand to evaluate different scenarios for improving the process design configuration

to assess the economic consequences of sparing equipment and flare limitations 


\subsubsection{General}

The field consists of producing wells being routed by ten remote manifolds to a centralised production station. The oil reservoir will be completed by artificial gas lifting and water injection. The oil is exported via a pipeline. The associated gas is offered to the government as part of the tax arrangements. From the manifolds the crude is transported to four main bulk separators in which the liquid is separated from the gas. The gas and liquid are treated separately by the gas system and the oil/water system respectively: In Figures 3 and 4 the process flow diagram is presented and discussed next.

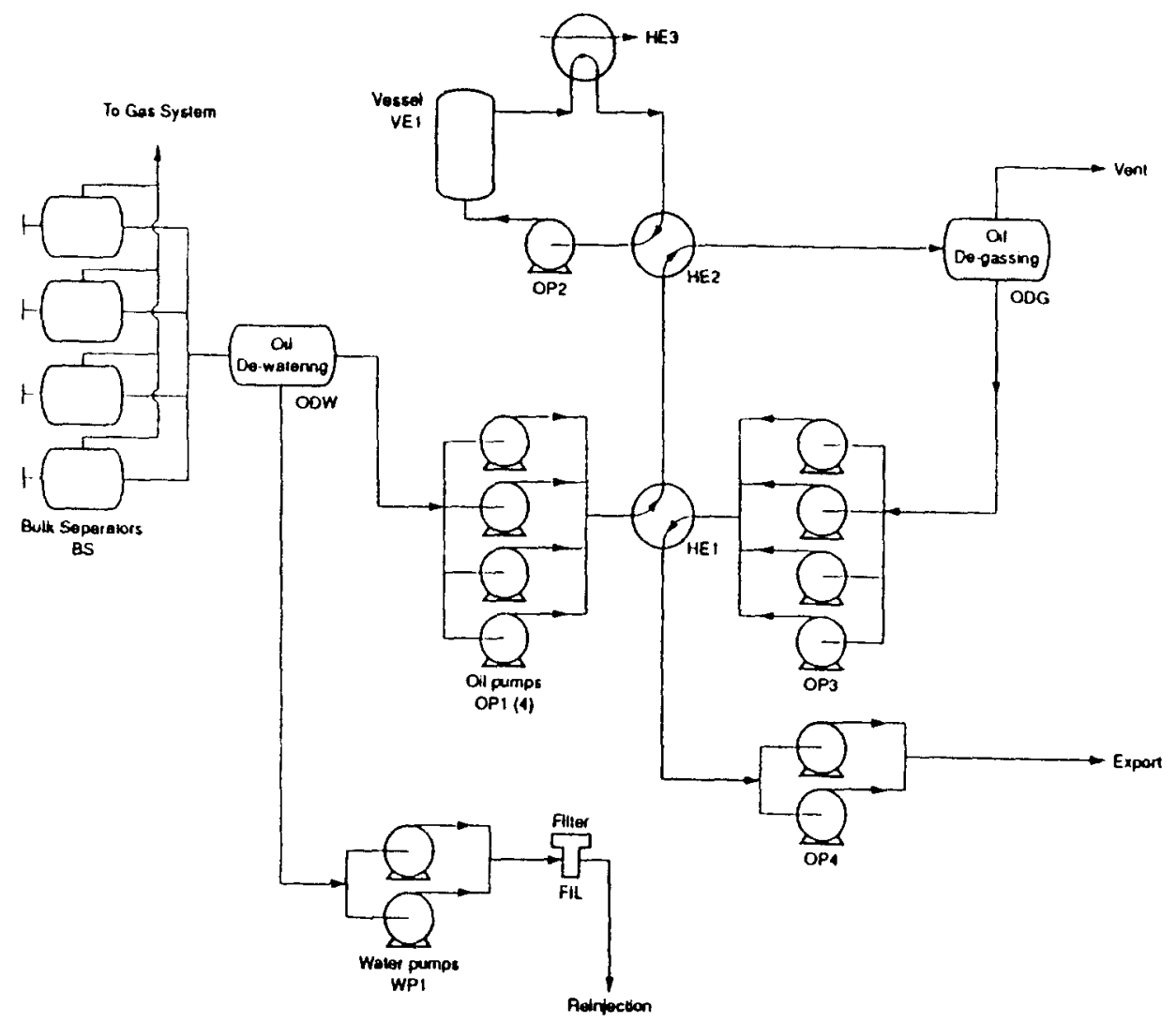

Figure 3: $\quad$ Process Flow Scheme Oil System

\subsubsection{Oil/water system}

The crude oil is separated from the water in the surge tank to be processed separately later. The water is separated from the oil for 


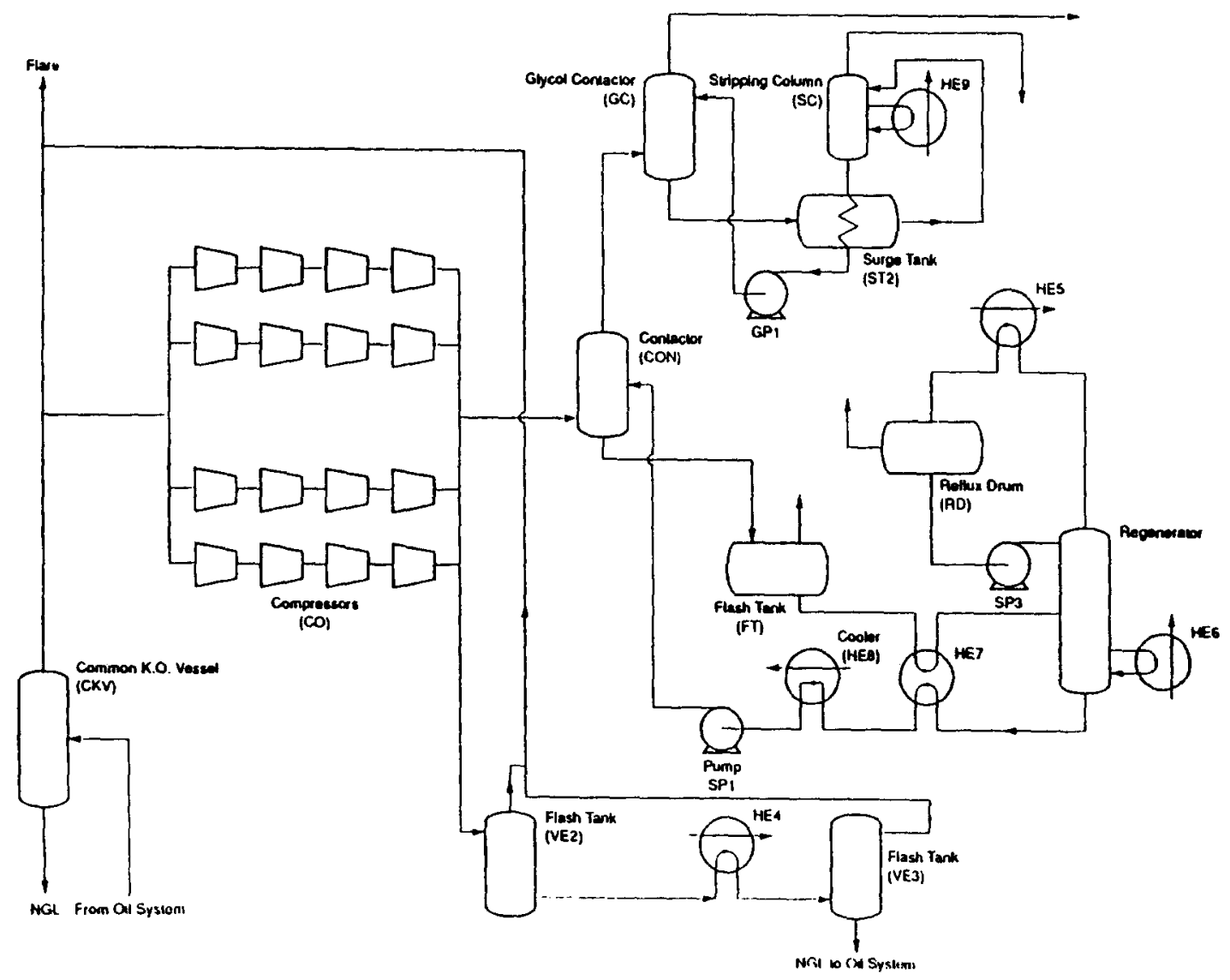

Figure 4: $\quad$ Process Flow Scheme Gas System

economic reasons (i.e. only the oil needs to be heated). The oil is heated (in the hot oil loop and by the returning hot oil flow) and de-gassed, after which the water and oil flow are combined again.

\subsubsection{Gas system}

The gas collected from the common knock-out vessels is compressed by four parallel trains. The NGL is collected via flash tanks after which it is added to the oil loop. The compressed gas is treated by the sulferox plant after which the gas is dehydrated to meet the required quality for export. Part of the gas is used for gas lift. The lift gas only needs to be compressed. When the treatment plants are down this lift gas can be by-passed to continue the oil production.

As a base case it will be assumed that the gas can be flared when 
the compression and/or the treatment plants are down.

\subsection{The reliability block diagram}

From the process flow diagram a reliability block diagram can be made that reflects the consequence of equipment failure(s) in the process system. For the reliability block diagram first the product needs to be specified. In principle for different products different reliability block diagrams need to be constructed. The diagram discussed next reflects the effectiveness of the oil production. For each component which may fail the consequence in oil production should be assessed.

In Figure 5 the reliability block diagram is presented and details are discussed next.

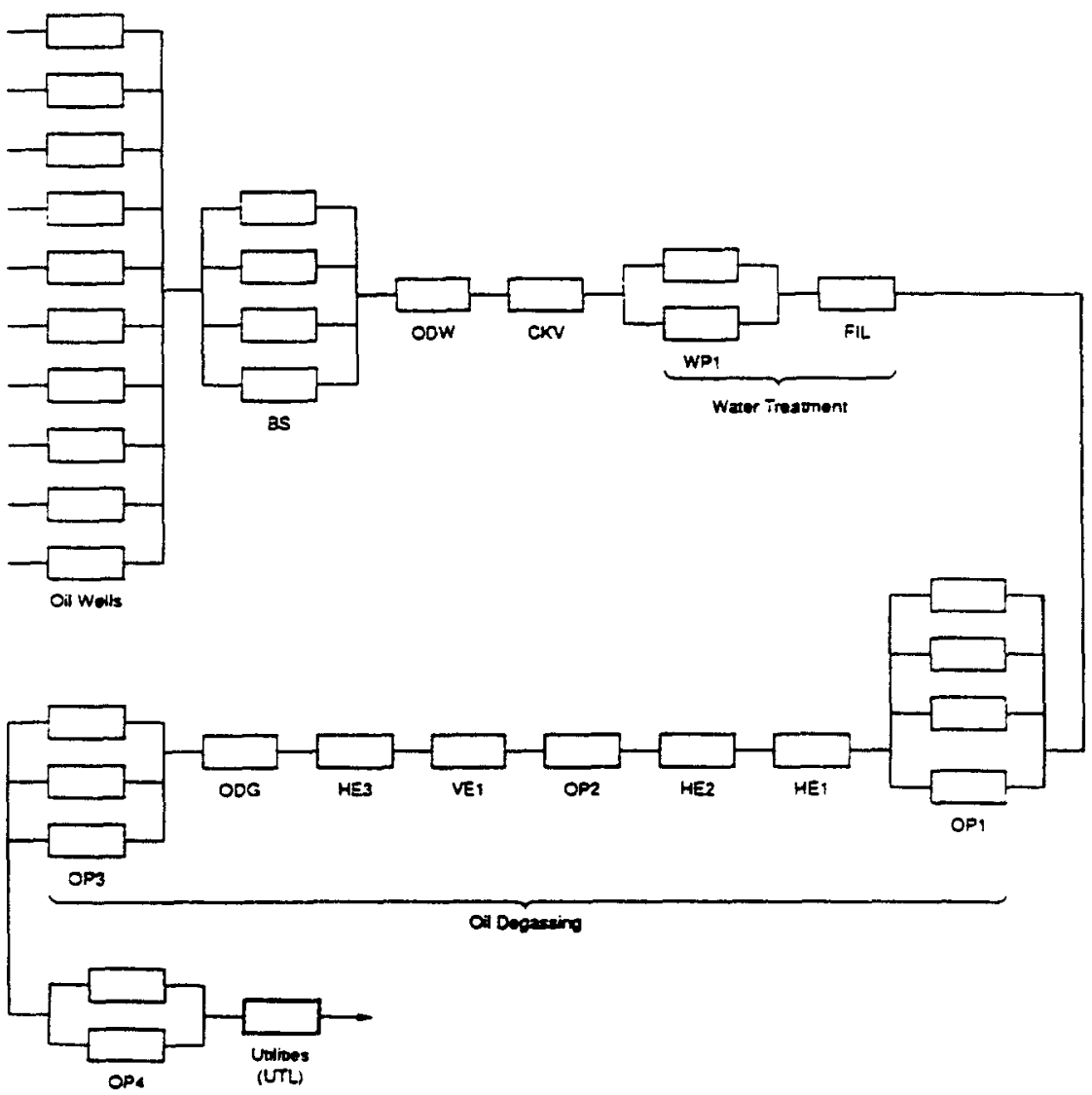

Figure 5: Reliability Block Diagram (Base Case) 


\subsubsection{Gas handling}

The wells, bulk separators (including the test separator), common knock-out vessel, gas compressors and gas treatment plant (sulferox, dehydration) are chained in series because if one of the units fails the oil production (partly) stops. The wet lift gas may by-pass the treatment plant if it fails (i.e., the oil production can continue). The capacity of the wet gas lift is $100 \%$ because the associated gas can be flared in the base case.

\subsubsection{Oil/water handling}

In the diagram the surge tank is put in series with the oil and water export facilities. The water pumps are chained in series with the oil facilities: if the water system fails the oil production is stopped (this is the shut-down philosophy).

\subsubsection{Utilities}

If the power generation and safety devices fail then the oil production stops. Therefore these are chained in series as well.

\subsubsection{Vessel inspections}

For the base case the main common vessels are not spared. Once in five years the complete plant is shut down for vessel inspections.

\subsection{Base case}

To improve the system effectiveness for the base case an option would be to spare equipment in order to make the system more robust towards breakdowns. From a criticality analysis the critical items within the network can be identified and sparing for these items may be considered as a solution.

For the improved (spared) system the system effectiveness is calculated, demonstrating that revenues from oil production have 
increased. On the other hand an investment will have to be made and also maintenance costs will increase. A present value cash flow analysis has to be performed to determine whether the increased revenues outweigh the extra costs involved.

\subsection{Flaring restrictions}

So far it has been assumed that, in case the compressors or gas treatment plants were down, all the gas could be flared. The volume of associated gas can be considered as a loss of gas. However, if the total volume to be flared is restricted then the effectiveness of the oil production is decreased. The operator will shut in oil wells when failure in the gas treatment occurs. This alternative operational strategy thus has an impact on the reliability block diagram; the revised diagram is depicted in Figure 6.

\subsection{Discussion}

The above presents a realistic, albeit relatively simple example of an availability assessment study. Analytical programs such as CREDO can analyse models with this complexity quite rapidly. In particular when a number of sparing options is to be investigated such speed may be quite essential. Also, what-if questions can be addressed on-line and the engineer operating the program may try to experimentally optimise the system guided by a combination of engineering judgement and quantitative results obtained from the program. This facilitates a 'reliability centred design' approach and leads to increased insight into the performance and sensitivities of the system.

\section{Open problems/opportunities}

Communication with consultants not always easy, amongst others because standard framework (terminology, way of modelling, etc.) is lacking. OREDA has opened up a wealth of information. It calls for 


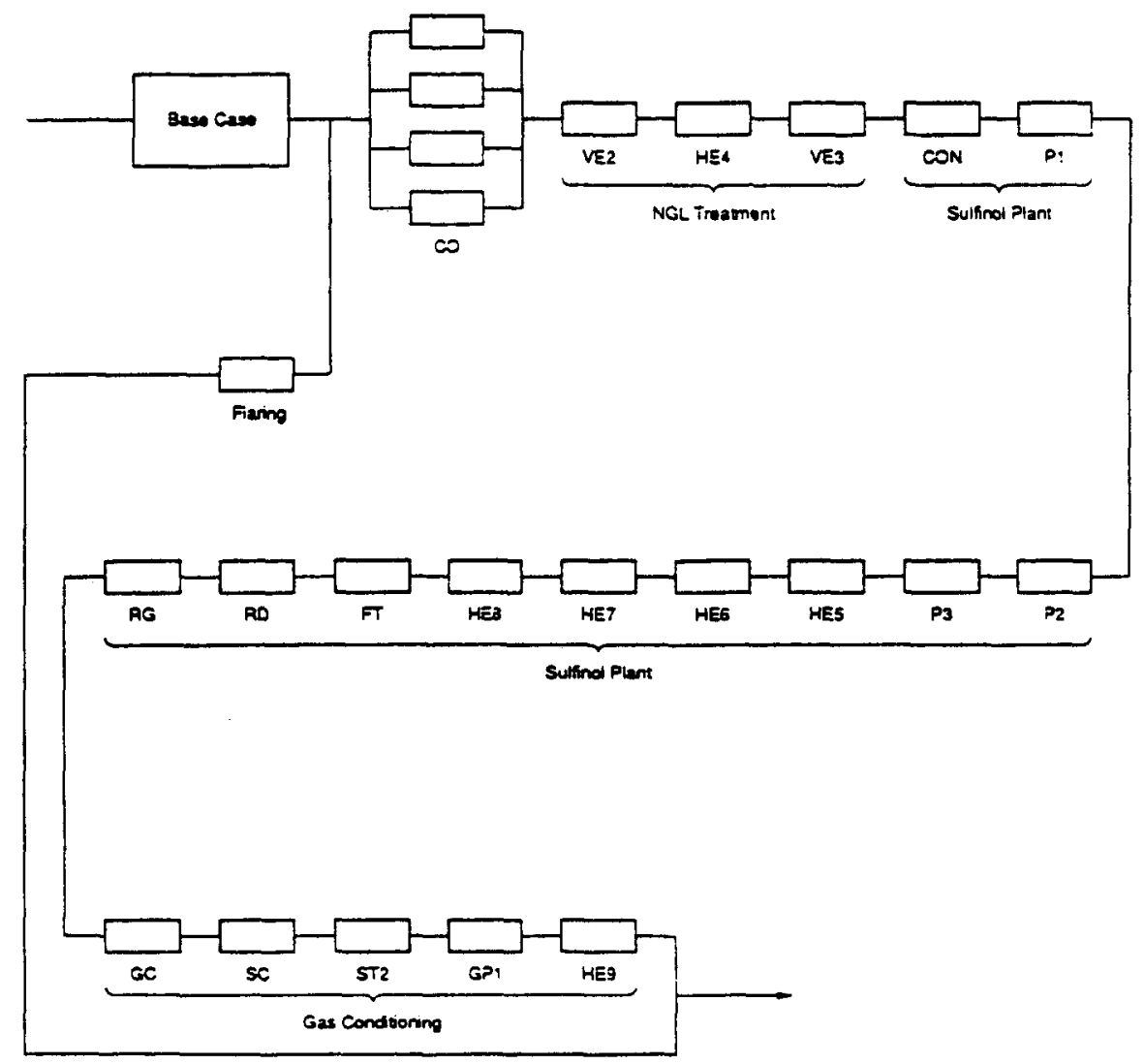

Figure 6: Reliability Block Diagram (Flaring Restriction)

analysis tools in particularly in the area of censored statistics. How to derive uncensored failure distributions from failures recorded under given maintenance regimes?). Preparations are being made to release student versions of both the PC-FOSP package and the OREDA database. The rationale behind this is that curricula's at Technical Universities in many instances cover reliability/availability analysis insufficiently. As a consequence, engineers usually have very little knowledge or even awareness of these topics, leading to missed opportunities for improved system design and operation.

There is a need for more advanced unit importance models to determine the criticality of process units both in terms of their reliability performance and throughput capacities. Existing models are too basic. Simulation programs should explain results and indicate which parameters 
are more important than others.

\section{Conclusions}

Although there has been some progress in availability assessment over the last years, the field is lacking behind other areas. We do see scope for tools based on analytical techniques, because they are faster and force the user to think about the modelling. The packages mentioned in this paper are able to tackle real problems. More research is needed enhance their application area. Apart from analytical methods we also see scope for simulation methods, but these should incorporate the latest simulation techniques, such as importance sampling, pertubation analysis and aggregation facilities.

\section{References}

Birolini, A. (1985), "On the use of stochastic processes in modeling reliability problems"., Springer Verlag, Berlin.

Brouwers, J.J.H. (1986), "Probabilistic descriptions of irregular system downtime", Rel. Eng., 15, p. 263-281.

Brouwers, J.J.H., Verbeek, P. and Thomson, W.A. (1986), "Analytical Systems Analysis Packagen", 9th ARTS., p.A/1/1-A/1/13, Nat. Centre. Syst. Rel., Warrington, UK.

Jardine, I.J.A. and Jackson, D. (1989), "Risk assessment and optimization of a safety system using the MAROS simulation package", Qual. Rel. Eng. Int., 5, p. 131-141.

Lie, C.H. Hwang, C.L. and Tillman, F.A. 11977), "Availability of maintained systems: a state -of-the-art survey", AllE Trans. p. 247-259.

OREDA (1992): "Offshore reliability data handbook 2 nd edition", published by the OREDA participants. Available from DnV Technica, P.O. Box 370, N-1322 Hovik, Norway.

Østebø, R., (1993), "Life cycle availability assessment in offshore field development", paper presented at Er. Saf. \& Rel. Conf. ESREL'93, München. 
Schmeiser, B., (1990), "Simulation experiments", in: Handbook of Operations Research, vol. 2, Stochastic Models, Heyman, D.P. and Sobel, M.J. ed., North-Holland, Amsterdam.

Van der Heijden, M.C. and Schornagel. A. (1988), "Interval uneffectiveness distribution for a k-out-of-n multistate reliability system with repair", Eur. Journ. Oper. Res., 36, p.

Van Rijn, C.F.H. and Schornagel, A. (1987), "Stamp, a new technique to assess the probability distribution of system effectiveness". The SRS Quart. Digest, Oct 1987, p. 50-62. 